\title{
Distribution of the coal flow in the mill-duct system of the As Pontes Power Plant using CFD modeling
}

\author{
J.L. Ferrín ${ }^{\text {a, } * \text {, L. Saavedra }}{ }^{b}$ \\ a Departamento de Matemática Aplicada, Universidad de Santiago de Compostela, 15782 Santiago de Compostela, Spain \\ ${ }^{b}$ E.T.S. Ingenieros Aeronáuticos, Pl. Cardenal Cisneros 3, Universidad Politécnica de Madrid, 28040 Madrid, Spain
}

Keywords:

Mill-ducts

Turbulent flow

CFD

Particle trajectories grid generation

\section{A B S T R A C T}

The efficiency of a Power Plant is affected by the distribution of the pulverized coal within the furnace. The coal, which is pulverized in the mills, is transported and distributed by the primary gas through the mill-ducts to the interior of the furnace. This is done with a double function: dry and enter the coal by different levels for optimizing the combustion in the sense that a complete combustion occurs with homogeneous heat fluxes to the walls. The mill-duct systems of a real Power Plant are very complex and they are not yet well understood. In particular, experimental data concerning the mass flows of coal to the different levels are very difficult to measure. CFD modeling can help to determine them. An Eulerian/Lagrangian approach is used due to the low solid-gas volume ratio.

\section{Introduction}

Coal fired boilers require accurate pulverized fuel flow measurement to balance coal mass distribution between burners. Balancing the coal mass improves the burner-to-burner stoichiometry, resulting in better plant performance and operating efficiency. In particular, regulation of the coal mass flows through the different levels of the burner allows optimizing the relation between the secondary air and the coal by each burner, which constitutes a key element in reducing $\mathrm{NO}_{x}$ emissions by allowing the optimization of the relationship between the secondary air and coal for each burner.

Non-homogeneous distribution of the coal particles within the millducts can lead to uneven wear of the ducting components. Uneven wear is biased toward the regions of higher coal flux and results in costly maintenance operations. In order to fine tune the oxygen supply to the furnace, a better understanding of the distribution of coal to the furnace, which is due to the complex mill ducting, is required. The number of techniques which can be utilized to measure coal flow is limited, generally expensive and does not readily adapt to fully operational industrial flows.

Typically, coal flow and fineness have been measured only at steady-state conditions and only intermittently using extractive sampling techniques. In order to obtain representative results, samples must be made in isokinetic conditions, i.e., maintaining identical suction velocities in the sample nozzles to the gas ones in the mill-duct. Representativeness of the samples is strongly influenced

\footnotetext{
* Corresponding author.

E-mail addresses: joseluis.ferrin@usc.es (J.L. Ferrín), laura.saavedra@upm.es (L. Saavedra).
}

by the location of the sampling plane. The high flow rates of coal common in the ducts introduce additional difficulties for obtaining representative samples.

Various extractive sampling methods are used for pulverized coal flow measurements (see Jensen and Elsen [1]). Systems like the "Rotorprobe", SMG10, ASME-probe, AKOMA and others are commonly used. All these systems have in common that they only provide snapshot type of information about coal flow. They require much manpower to use, especially if one wishes to measure several pipes at the same time. Extractive coal flow measurements remain a difficult and dirty job. The extractive methods also require steady state conditions during the sampling phase, meaning that the pulverizer being measured is placed in manual control precluding gaining knowledge of what is happening to coal flow during load changes.

In recent years systems for real time measurements of flow and particle size distribution in pulverized coal furnaces have been developed. For example, Electric Charge Transfer (ECT) systems provide boiler operators with continuous on-line indication of coal flow distribution, conduit velocity and particle fineness. This technology measures electric charges present in the two phases of the flow transportation and uses these signals to determine flow characteristics. Some reviews have been dedicated to on-line, continuous and non-invasive techniques for the measurement of mass flow rate of solids in pneumatic conveying pipelines (see, for example, Yan [2], Arakaki et al. [3], Ahmed and Ismail [4] or Zheng and Liu [5]).

Distribution of coal and gas mixture within complicated mill-ducts has been investigated experimentally and numerically to better understand the flow patterns (see, for example, Shah et al. [6], Dodds et al. [7], Vuthaluru et al. [8,9] and Arakaki et al. [10]). CFD analysis is used to analyze flow profiles and design/redesign ducts to improve overall 


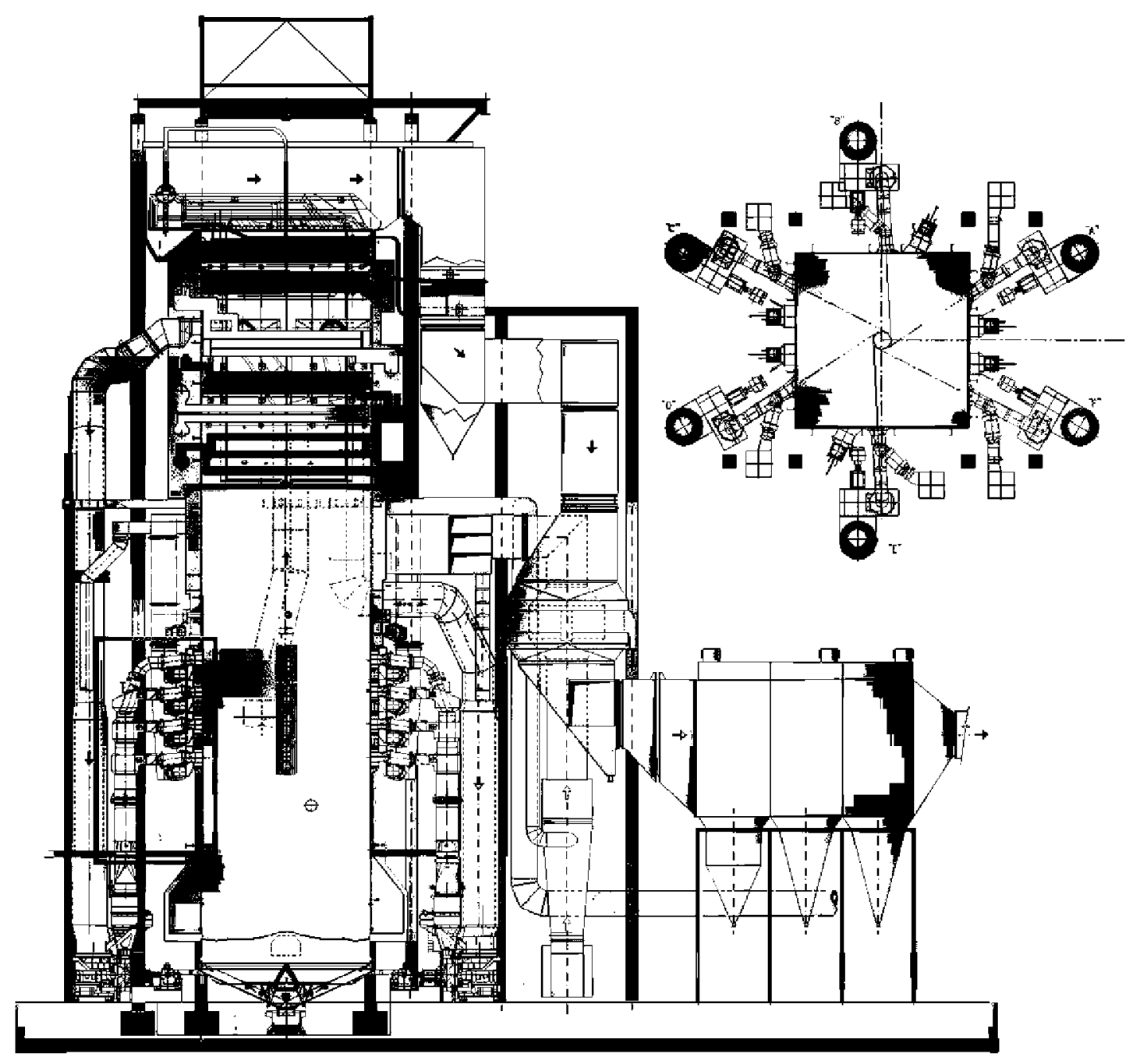

Fig. 1. Furnace of the As Pontes Power Plant.

performance. Full scale model fabrication and certified wind tunnel testing are used to develop application specific products that will measure accurately where no standard flow measurement can.

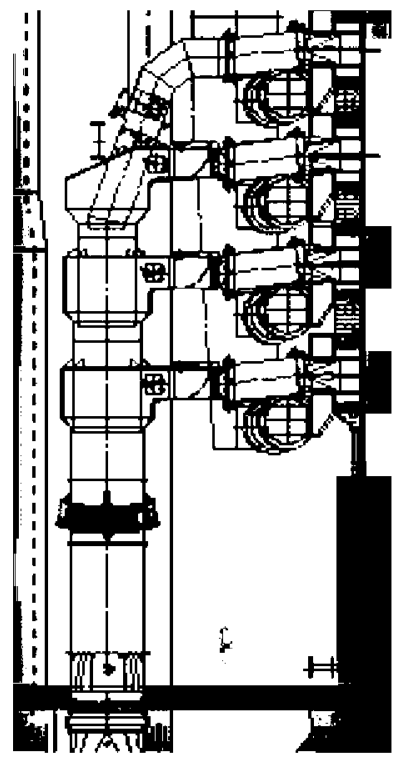

Fig. 2. Detail of the air ducts.
The aim of this paper is to better understand the distribution of coal flow within the mill-ducts of an operating real-life power station (U.P.T. As Pontes, Spain) by numerical methods. CFD modeling of the mill-duct gas and coal flows is compared with experimental data obtained with manual methods in this particular plant.

Table 1

Results of the characterization of the grinding system.

\begin{tabular}{|c|c|c|c|c|c|c|c|c|}
\hline \multirow[t]{2}{*}{$\begin{array}{l}\text { Position of the } \\
\text { concentrator }\left({ }^{\circ}\right)\end{array}$} & \multicolumn{4}{|c|}{$\begin{array}{l}\text { Mass of gas in mill fingers } \\
(\%)\end{array}$} & \multicolumn{4}{|c|}{$\begin{array}{l}\text { Mass of coal in mill fingers } \\
(\%)\end{array}$} \\
\hline & 1 & 2 & 3 & 4 & 1 & 2 & 3 & 4 \\
\hline-8 & 21.32 & 21.37 & 26.17 & 31.14 & 33.23 & 30.00 & 21.18 & 15.59 \\
\hline-8 & 19.88 & 20.65 & 24.70 & 34.77 & 31.60 & 28.77 & 22.44 & 17.19 \\
\hline-5 & 20.46 & 20.73 & 24.87 & 33.94 & 34.25 & 30.10 & 20.88 & 14.77 \\
\hline-5 & 21.06 & 19.86 & 25.50 & 34.58 & 31.67 & 31.70 & 23.15 & 13.48 \\
\hline 0 & 21.33 & 20.26 & 25.12 & 33.29 & 29.36 & 41.48 & 18.65 & 10.51 \\
\hline 0 & 20.08 & 19.78 & 24.24 & 35.90 & 34.75 & 37.73 & 19.71 & 7.81 \\
\hline 5 & 19.64 & 19.63 & 24.70 & 36.03 & 34.85 & 44.97 & 14.61 & 5.57 \\
\hline 5 & 19.82 & 19.77 & 25.03 & 35.38 & 39.15 & 36.95 & 16.38 & 7.52 \\
\hline 10 & 19.33 & 20.02 & 25.59 & 35.06 & 44.80 & 37.34 & 13.08 & 4.78 \\
\hline 10 & 20.44 & 20.17 & 24.18 & 35.21 & 43.39 & 39.67 & 12.15 & 4.79 \\
\hline 0 & 19.77 & 19.41 & 25.18 & 35.64 & 33.99 & 37.72 & 17.43 & 10.86 \\
\hline-5 & 19.90 & 20.37 & 24.87 & 34.86 & 35.27 & 27.43 & 21.18 & 16.12 \\
\hline 0 & 20.20 & 21.34 & 26.04 & 32.20 & 31.05 & 37.67 & 20.83 & 10.45 \\
\hline-5 & 20.47 & 22.12 & 24.92 & 32.44 & 31.20 & 27.05 & 22.46 & 19.29 \\
\hline-8 & 20.09 & 22.16 & 24.51 & 33.22 & 29.96 & 24.55 & 23.04 & 22.46 \\
\hline
\end{tabular}


Table 2

Data of the gas mixture at the mill outlet.

\begin{tabular}{ll}
\hline Composition & $(\%)$ \\
\hline $\mathrm{CO}_{2}$ & 16.413 \\
$\mathrm{H}_{2} \mathrm{O}$ & 10.058 \\
$\mathrm{~N}_{2}$ & 66.596 \\
$\mathrm{SO}_{2}$ & 0.015 \\
$\mathrm{O}_{2}$ & 6.918 \\
Temperature & $220^{\circ} \mathrm{C}$ \\
Density & $0.7 \mathrm{~kg} / \mathrm{m}^{3}$ \\
Mass flow rate & $44.36 \mathrm{~kg} / \mathrm{s}$ \\
\hline
\end{tabular}

This paper is organized as follows. The geometry, the methodology used to obtain the experimental data and the operating conditions of the furnace are described in Section 2. The computation domain used in the simulations as well as the mathematical model used are described in Section 3. Finally Section 4 shows the numerical results. They are compared with the experimental measures, showing a good agreement among the gas flows but not so good with respect to coal particle ones. We finish with some conclusions.

\section{Description of the installation}

\subsection{Geometry}

The tangentially fired furnaces of As Pontes Power Plant have six mills and six burners and each burner is divided into four levels each one consisting of one gas/coal inlet and three air inlets. In Fig. 1 a plane of the furnace considered in this paper is shown.

In the mills the coal is pulverized to improve the effectiveness of the combustion and then it is dragged into the boiler by a gas stream. This gas mixture has a low oxygen content in order to avoid the coal combustion to start before it goes into the fireplace. The mixture of recirculation gases and pulverized coal goes from the mills to the chamber through a set of ducts called windbox. This windbox is divided into four ducts which distribute the gas/coal mixture to the four levels of each burner. Inside the windbox is located the concentrator, a piece formed by eight directional vanes that can adopt different angles; depending on the position of such vanes the distribution of the coal changes giving priority to some levels. Fig. 2 shows the mill-ducts of the burner (the zone rounded by a box in Fig. 1) that we are going to simulate. The diameter of the central duct is $1.95 \mathrm{~m}$ near the base, and $1.13 \mathrm{~m}$ on the top.

\subsection{Methodology for the measurements}

In order to characterize the grinding in the mill of a windbox of the Power Plant of As Pontes, the mass flow in the fingers of the mill D in Group 3 has been determined. For that reason, two measurement campaigns including all the activities involved in the manual characterization of the mass flow rates as well as sampling of pulverized coal particles for the analysis of their size. These activities have been carried out under normal load conditions ( $32 \mathrm{t} / \mathrm{h}$ of coal and $520 \mathrm{rpm}$ of the wheel mixer) and different positions of the concentrator. In the first campaign, carried out in March 2007, two tests have been conducted for each of the five conditions characterized (position of the concentrator to $-8^{\circ},-5^{\circ}, 0^{\circ},+5^{\circ}$ and $+10^{\circ}$ ), and two additional contrast tests with the positions of $0^{\circ}$ and $-5^{\circ}$, to confirm repeatability of results. In the second campaign, carried out in October 2007, only one test for the positions $-8^{\circ},-5^{\circ}$ and $0^{\circ}$, has been conducted.

Table 3

Data of coal at the mill outlet.

\begin{tabular}{ll}
\hline Density & $922 \mathrm{~kg} / \mathrm{m}^{3}$ \\
Mass flow rate & $6.636 \mathrm{~kg} / \mathrm{s}$
\end{tabular}

Table 4

Rosin-Rammler parameters for the different experiments.

\begin{tabular}{lccccc}
\hline Position (angle) & -8 & -5 & 0 & 5 & 10 \\
Spread parameter & 0.9905 & 0.9660 & 0.9793 & 1.0060 & 0.9900 \\
Medium diameter $(\mu \mathrm{m})$ & 157.02 & 143.26 & 146.55 & 153.50 & 152.95 \\
\hline
\end{tabular}

Next we will describe the methodology used in the coal flow determination through the fingers of the mill $\mathrm{D}$ for the group 3 . These determinations are used to obtain the individual carbon contributions for each of the fingers and test conditions (different positions of the concentrator).

The measures involved were made by using an equipment of manual isokinetic sampling, owned by the Power Plant of As Pontes, for measures of dynamic and static pressures in ducts, temperature and mass flows of pulverized coal. This sampling equipment is designed for use together with a zero pressure probe, which allows the imposition of isokinetic conditions during the extraction of the coal samples. It is well-known that, although this procedure can measure coal mass fluxes within power station mill-ducts, these measurements are strongly dependent on the milling conditions.

For the determination of dynamic and static pressures in each finger of the mill, four points for each of the four extraction positions

\section{(a) Computational domain}

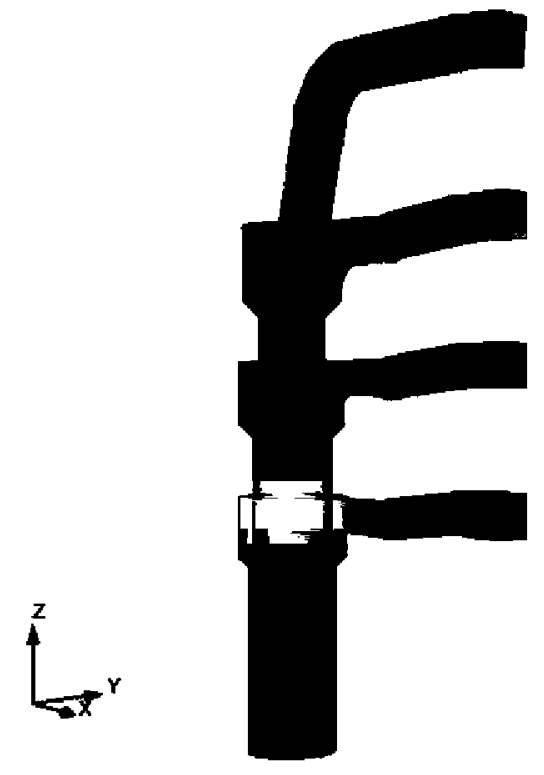

(b) Detail of the concentrator

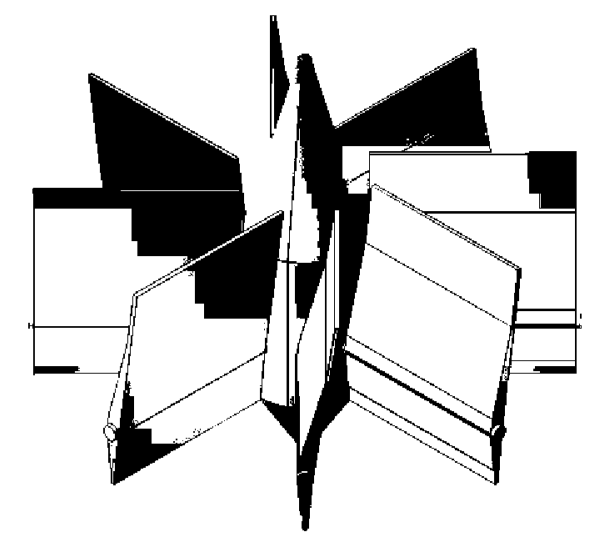

Fig. 3. CAD of the mill-duct. 
(a) Angle $-8^{\circ}$

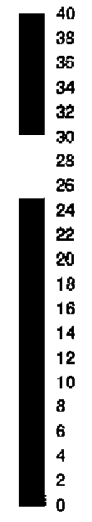

(b) Angle $-5^{\circ}$

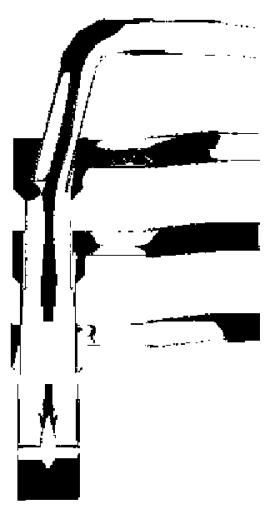

(d) Angle $5^{\circ}$

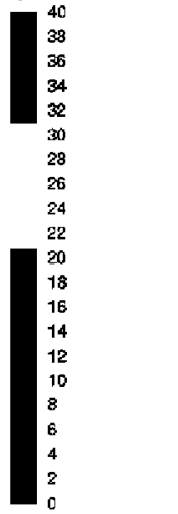

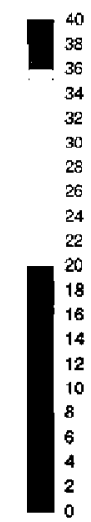

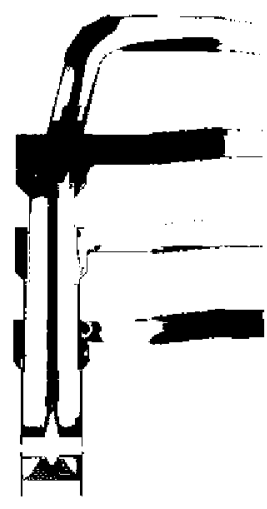

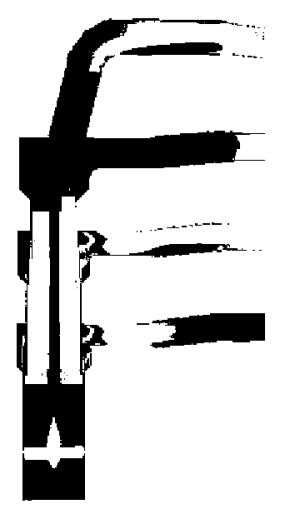

(e) Angle $10^{\circ}$

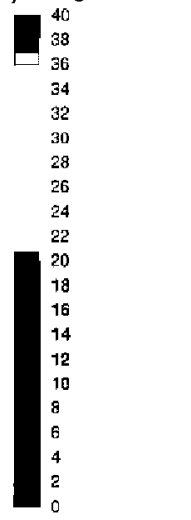

(c) Angle $0^{\circ}$

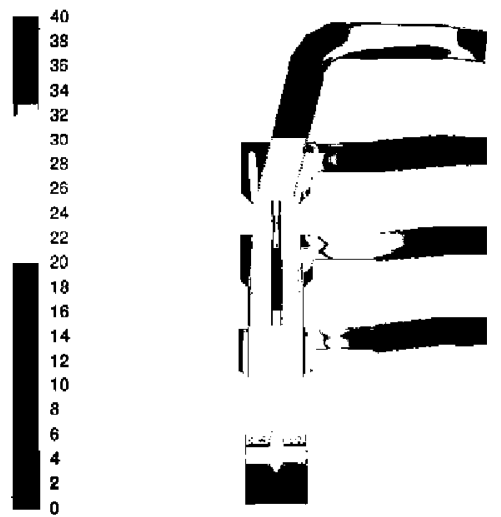

Fig. 4. Contours of the velocity $(\mathrm{m} / \mathrm{s})$ in the plane $Y=0$ according to the concentrator position.

in each one of the lower fingers and ten points for each of the four diameters available in the upper finger have been considered, using zero-pressure probes with different lengths and the Magnehelic (pressure measurements) integrated in the portable equipment of isokinetic sampling. It assures isokinetic conditions by continuous measurement of pressure in the duct. This sampling was conducted in each of the four extraction positions in each finger, by a complete scanning of its section for $2 \mathrm{~min}$. Thus, the four samples taken in each finger mill have been composed as a single representative one, which has been weighted in the field and was sent to the laboratory for the sieve analysis. This provides coal sizing and moisture content. In order to prevent jams caused by coal particles, or possible condensation of gases in the measuring circuit, the blow-off of the whole line after measuring the pressure in each of the four extraction positions of each finger has been carried out.

The main results of each of these tests are included in Table 1 where, for each scenario tested, the control signals that determine the operating conditions as well as the mass flows of gas and coal obtained and their percentage distribution obtained by each finger are collected.

\subsection{Operating conditions}

The gases at the outlet of the mills are a mixture of hot recirculation gases, cold recirculation gases, fresh air infiltrated to the mills and water vapor from the moisture evaporation of the coal that occurs during its pulverization. The exact composition and temperature of the gas mixture are known and only vary slightly from the mills to its entry into the burner. In Table 2, the measurements of the density and the temperature of the gas mixture inside the mill-ducts can be seen.

The coal used in the power plant is a subbituminous coal trademarked as Envirocoal from the Indonesian company Adaro Energy. As is assumed that the temperature and mass of the coal particles remain constant during all the processes, we only need to know the mass flow rate of the coal supplied and its density; this values can be seen in Table 3 . The diameters of the coal particles are between $10 \mu \mathrm{m}$ and $1000 \mu \mathrm{m}$. After the pulverization of the coal in the mills, the data from the particle size distribution is extracted and further approximated using a Rosin-Rammler distribution function. These data are different in each test done in the plant for each position of the concentrator. Therefore, in order to compare the numerical results with all the measurements done, we will consider the mean values of the Rosin-Rammler parameters of all the tests done for each position. So, for the initial size distribution of the particles, we use these mean values that can be seen in Table 4 for each angle of the concentrator.

\section{Numerical simulation}

In any CFD modeling work, three stages must be carried out: geometry creation, grid generation and flow simulation. In the present paper CATIA v5 was used for the first one, ANSYS MESHING for the second one and ANSYS FLUENT 13.0 for the last one.

\subsection{Computational domain and meshes}

The computational domain is approximately $15 \mathrm{~m}$ high and the horizontal tubes are $8 \mathrm{~m}$ long. Fig. 3(a) plots the geometry of the mill-ducts and the concentrator corresponding to the position $-8^{\circ}$. A more detailed view of the concentrator can be seen in Fig. 3(b).

The computational domain is different depending on the position of the concentrator, so five different meshes have been done. For each position a tetrahedral unstructured mesh having approximately six 
(a) Angle $-8^{\circ}$

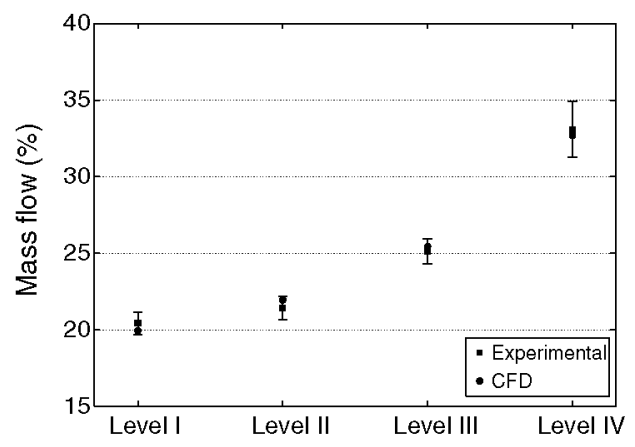

(c) Angle $0^{\circ}$

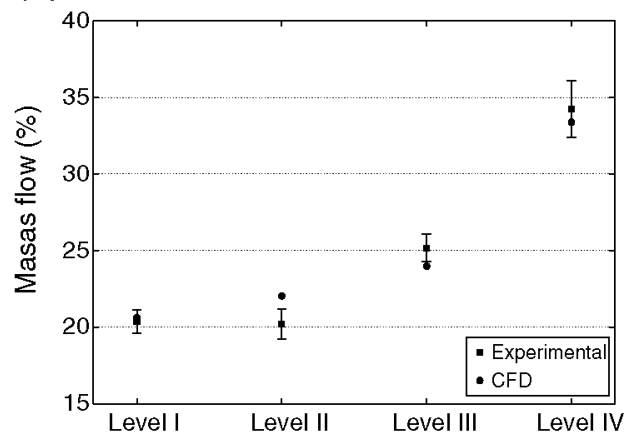

(b) Angle $-5^{\circ}$

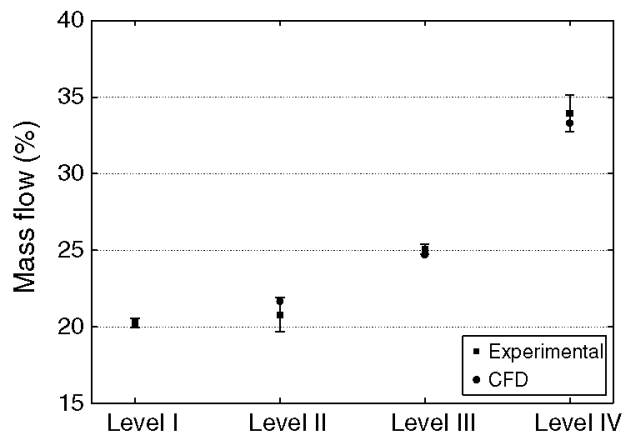

(d) Angle $5^{\circ}$

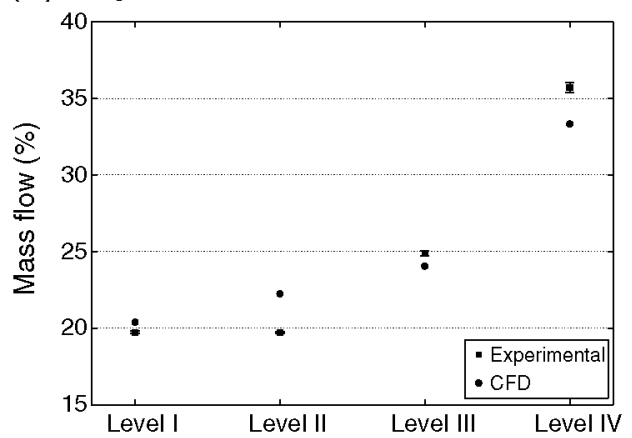

(e) Angle $10^{\circ}$

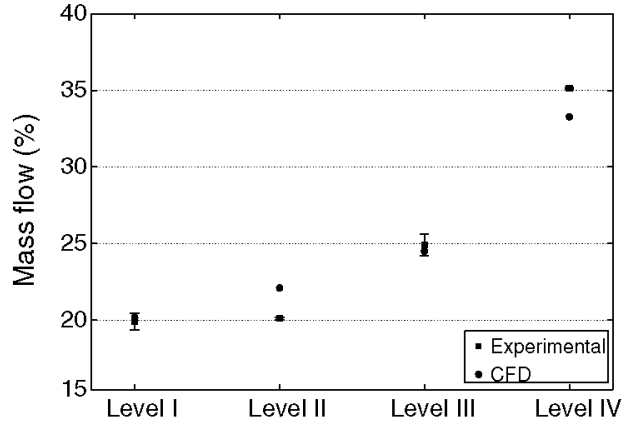

Fig. 5. Distribution of the gas mass flow rate according to the concentrator position.

millions of cells is found to provide good resolution and grid independent results.

\subsection{Mathematical modeling}

In this section we present the mathematical models for obtaining the results for the gas and pulverized coal particle flows through the mill-duct system.

We want to predict a multiphase flow consisting of a turbulent gas flow-field and dispersed solid particles. The first one is a mixture of non-reactive gases whose composition, temperature and viscosity remain constant from the outlet of the mill to the inlet of the boiler. On the other hand, the solid phase is formed by pulverized coal particles having an almost insignificant volumetric fraction thus their effects on gas flow momentum and particle collisions can safely be neglected. The humidity of coal particles is released in the mills and the gases dragging them have a small content of oxygen and such a low temperature that neither gasification reactions nor volatile release can occur during the transport of the particles within the mill-ducts; so throughout the whole process we can consider the coal particles as inert particles. Therefore, we can consider that the only influence of the gas phase over the solid phase is that the coal particles move due to the gas velocity and they are slightly heated or cooled by heat transfer between the gases and the particles, so this last phenomenon is not taken into account in our simulations. Keeping these ideas in mind, the gas phase can be considered uncoupled from the dispersed phase and we can solve the gas flow field until it achieves the steady state and then compute the trajectory of the coal particles and obtain its distribution.

\subsubsection{Gas phase}

Although the gas phase is formed by a mixture of gaseous species as its temperature and density remain constant, while there may be minor changes in its composition, it can be modeled as a single gas flow with constant density. For this reason, to calculate the continuous gas phase the incompressible Navier-Stokes equations can be used.

We will use for the treatment of the turbulence a RANS approximation and to close the terms in the averaged Navier-Stokes equations the realizable $k-\epsilon$ model proposed by Shih et al. [11]. This model provided better performance than the standard version for separated flows and flows with secondary flow features. Furthermore, the particle dispersion model described later needs the values of $k$ and $\epsilon$, so that we are 
(a) Angle $-8^{\circ}$

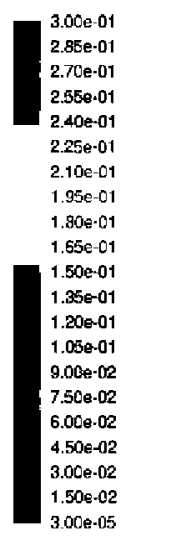

(b) Angle $-5^{\circ}$

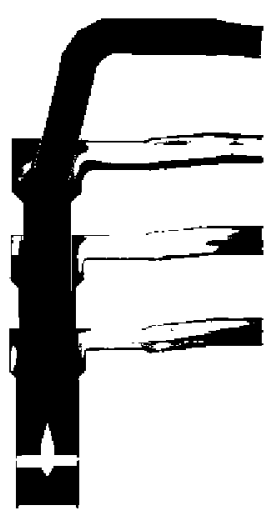

(d) Angle $5^{\circ}$

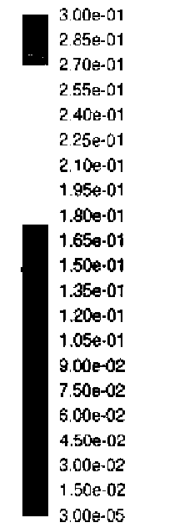

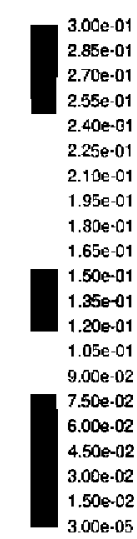

(c) Angle $0^{\circ}$
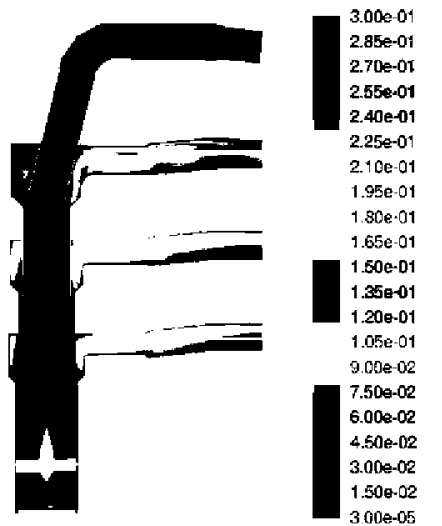

(e) Angle $10^{\circ}$

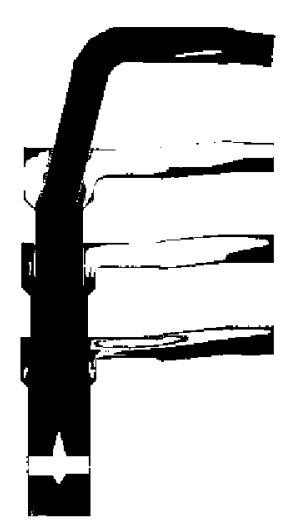

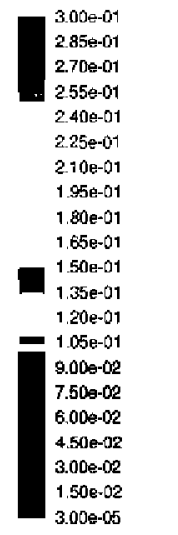

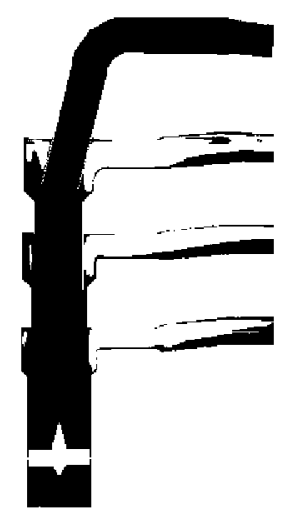

Fig. 6. Contours of the turbulent viscosity $(\mathrm{kg} / \mathrm{m} \mathrm{s})$ in the plane $Y=0$ according to the concentrator position.

forced to choose a turbulence model that provides values for these variables.

\subsubsection{Solid phase}

Since a Lagrangian description for the analysis of the coal particles behavior is used, the trajectory of each single particle throughout the computational domain must be calculated. For particles much heavier than the carrier fluid $\left(\rho_{p} \gg \rho_{g}\right)$ a realistic approximation is to consider that the aerodynamic drag and the gravity are the predominant forces acting on the pulverized coal particles transported in the mill-ducts, although other forces may also have an influence on the trajectory of the particles. Therefore, the particle velocity can be obtained by solving the initial value problem:

$\frac{d \mathbf{v}_{p}}{d t}=F_{A}\left(\mathbf{v}_{g}-\mathbf{v}_{p}\right)+\mathbf{g}$

$\mathbf{v}_{p}(0)=\mathbf{v}_{p 0}$

where $F_{A}\left(\mathbf{v}_{g}-\mathbf{v}_{p}\right)$ is the drag force per unit mass and

$F_{A}=\frac{3}{4} \frac{\mu}{\rho_{p} d_{p}^{2}} C_{D} R e$

Here $\mathbf{v}_{g}$ is the gas mixture velocity, $\mathbf{v}_{p}$ the particle velocity, $d_{p}$ the diameter of the particle, $\mu$ the gas viscosity, and Re the Reynolds number relative to the particle,

$R e=\rho_{g}\left\|\mathbf{v}_{g}-\mathbf{v}_{p}\right\| \frac{d_{p}}{\mu}$ and $C_{D}$ is the drag coefficient given by the Schiller-Naumann model (see Schiller and Naumann [12])

$C_{D}=\frac{24}{R e}\left(1+0.15 R e^{0.687}\right)$

The governing equation for particle location $\mathbf{x}_{p}$ is

$\frac{d \mathbf{x}_{p}}{d t}=\mathbf{v}_{p}$

The particle motion model given by Eqs. (1)-(6) needs the instantaneous local value of the velocity of the gas mixture at $\mathbf{x}_{p}, \mathbf{v}_{g}\left(\mathbf{x}_{p}, t\right)$, known as the fluid velocity "seen" or sampled by the particle as it moves across the flow. Clearly, modeling this term would not be necessary if the gas phase was fully resolved but for our problem a DNS approach is not feasible. In the context of RANS, the instantaneous value of the gas velocity is obtained by coupling this particle motion model with a stochastic model to take into account the particle dispersion; for example, a discrete random walk model (DRW). The "seen" velocity is decomposed as $\mathbf{v}_{g}=\overline{\mathbf{v}}_{g}+\mathbf{v}_{g}$, with $\overline{\mathbf{v}}_{g}$ the mean fluid velocity at the particle location determined by the mean Navier-Stokes equations and $\mathbf{v}_{g}$ the fluctuating velocity which has to somehow be reconstructed from the mean variables of the problem $\left(\overline{\mathbf{v}}_{g}, k\right.$ and $\left.\epsilon\right)$. DRW models compute the particle dispersion with random sampling to obtain instantaneous flow properties for a statistically significant number of particle trajectories. Each trajectory calculated for each particle size and initial location is called a "try". The model selected in this paper considers a Gaussian distributed random velocity fluctuation with zero mean and variance related 
(a) Angle $-8^{\circ}$

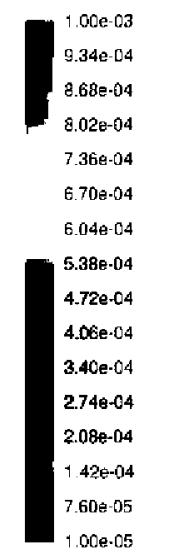

(c) Angle $0^{\circ}$

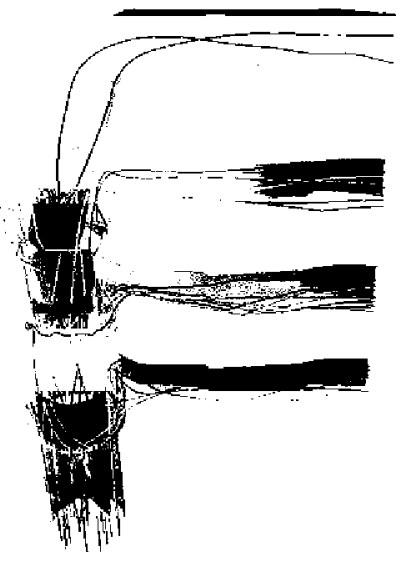

(b) Angle $-5^{\circ}$

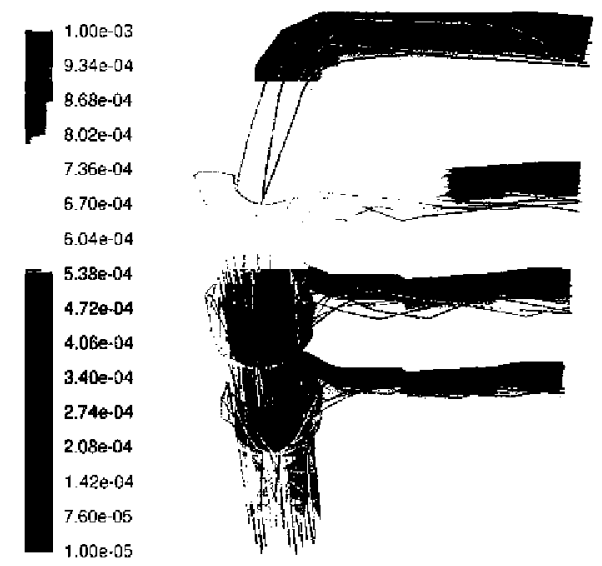

(d) Angle $5^{\circ}$
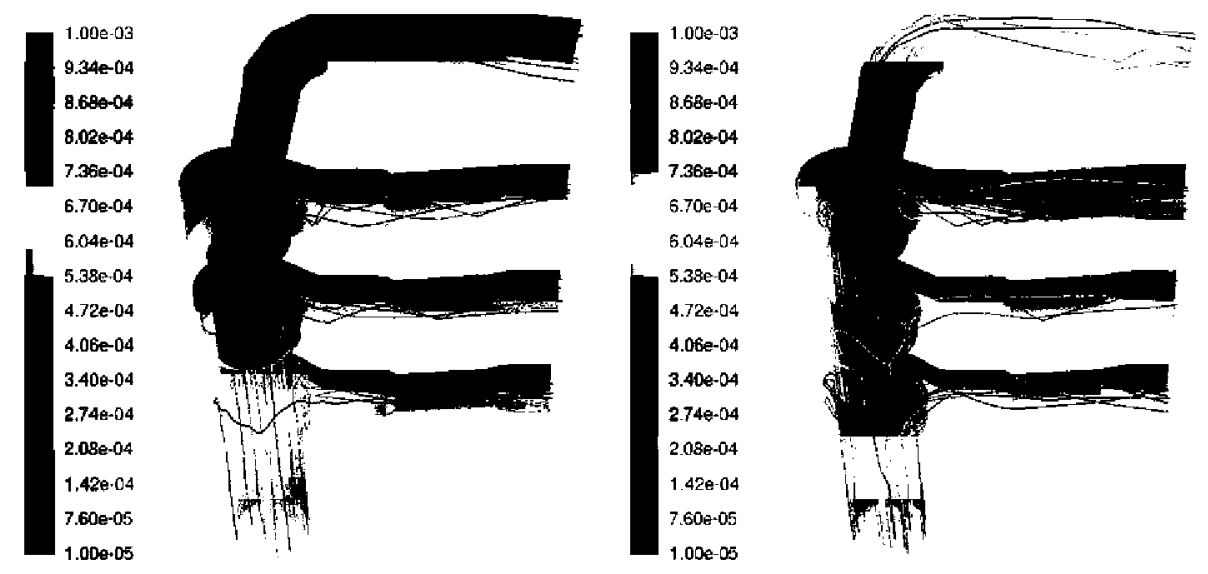

(e) Angle $10^{\circ}$

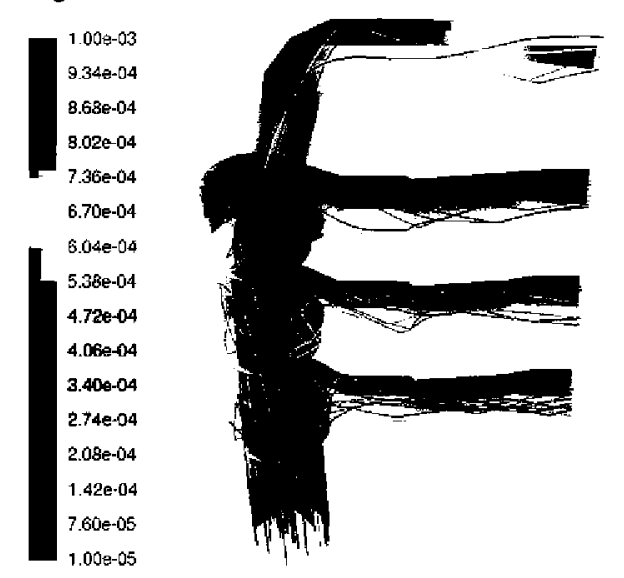

Fig. 7. Trajectories of the coal particles colored by its diameter according to the concentrator position.

to the turbulent velocity scale computed from the $k-\epsilon$ model. So, the fluctuating value of the velocity is given by

$\mathbf{v}_{g}=\left(\xi_{1} \sqrt{\frac{2 k}{3}}, \xi_{2} \sqrt{\frac{2 k}{3}}, \xi_{3} \sqrt{\frac{2 k}{3}}\right)$

where $\xi_{i}, i=1,2,3$ being normal distributed random numbers. A new fluctuation of the velocity is computed when the characteristic lifetime of the eddy or the characteristic time required for the particle to cross the eddy, is elapsed.
This model is based on the eddy-particle interaction model introduced by Gosman and Ioannides [13]. Compared with many random walk models, this model produces reasonably good behavior for flows with homogeneous turbulence but may give non-physical results in strongly inhomogeneous diffusion-dominated flows. The suitability of many other random walk models and particle dispersion models was analyzed in $[14,15]$.

Different stochastic models based on the Langevin equation, in the framework of PDF modeling of dispersed two-phase turbulent flows, can be seen in Pozorski and Minier [16,17]. More recently, the work 
(a) Angle $-8^{\circ}$

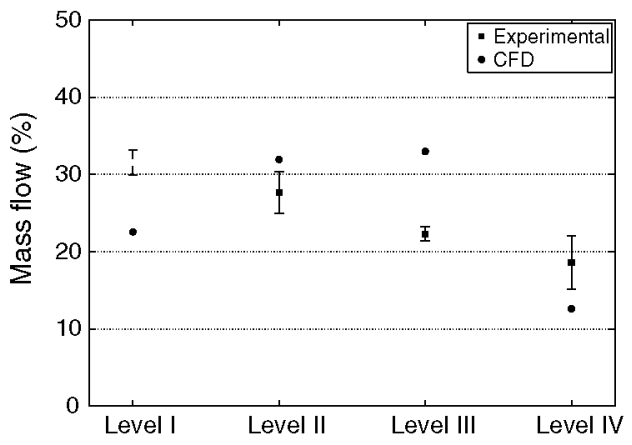

(c) Angle $0^{\circ}$

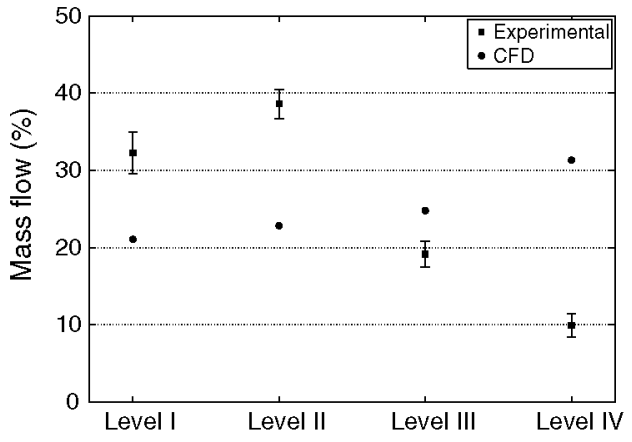

(b) Angle $-5^{\circ}$

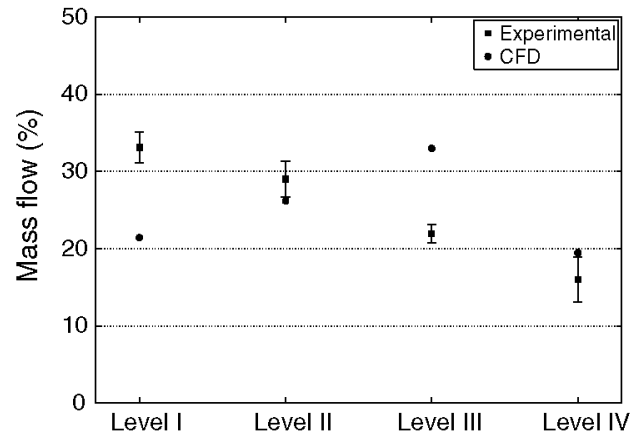

(d) Angle $5^{\circ}$

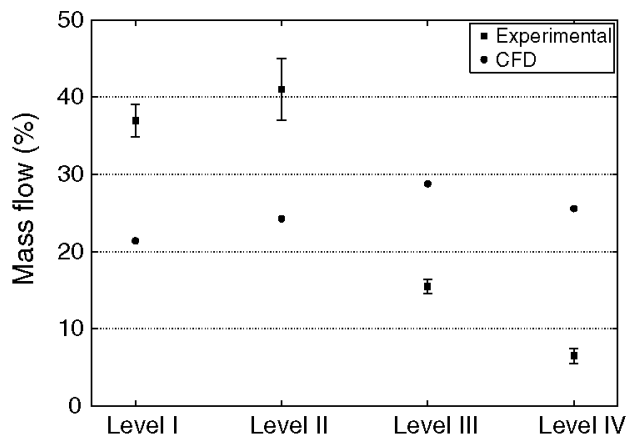

(e) Angle $10^{\circ}$

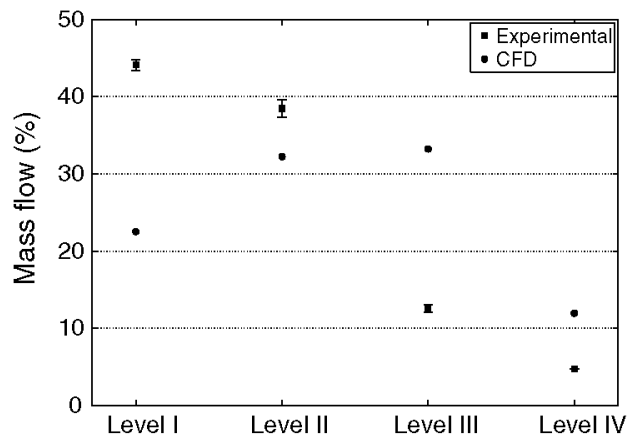

Fig. 8. Distribution of the coal mass flow rate according to the concentrator position.

done by Peirano et al. (see, for example, [18]) shows a general numerical approach and how PDF models, Langevin stochastic equations, particle/mesh and dynamical Monte Carlo methods are connected. In these models stochastic differential equations have to be solved to obtain the values of the "seen" fluid variables and the description of the dispersion of the particles is more accurate but they are not yet implemented in the software used in this paper.

\subsubsection{Boundary conditions}

For the gas inlet we use a uniform mass flow inlet boundary condition. The $k$ and $\epsilon$ values at the inlet are computed using the turbulence intensity and the hydraulic diameter taking into account the experimental data related to the velocities and the dimensions of the inlet that can be seen in Table 2. Assuming that the gas reaching the concentrator is fully-developed, the turbulence intensity can be estimated using the following empirical formula (valid for the core of a fully-developed duct flow)

$$
I=0.16(\operatorname{Re})^{-1 / 8}
$$

The Reynolds number at the inlet of the mill-ducts is about $1.68 \times 10^{6}$, so we can set $I=2.66 \%$ and $k$ and $\epsilon$ are computed using the formulas

$k=\frac{3}{2}(\overline{\mathbf{v}} I)^{2}, \quad \epsilon=C_{\mu}^{3 / 4} \frac{k^{3 / 2}}{0.07 D}$,

with $D$ the diameter of the inlet duct, leading to $k=0.51$ and $\epsilon=0.43$.

The outlets, which are the boiler inlets, are defined with a pressureoutlet boundary condition with a constant value for the pressure slightly lower than the atmospheric pressure. This is done because the boiler operates at a slightly negative pressure to reduce the possibility of outward flow through inspection doors, when these are opened.

Finally, at the walls we impose the non-slip condition for the gaseous flow. For the solid phase we must define the behavior of the particles when they impact with a wall. We use a model for the interaction between wall and solid particles known as hard sphere model (see for instance Mitarai and Nakanishi [19]). This model can also be used to 
(a) Level I

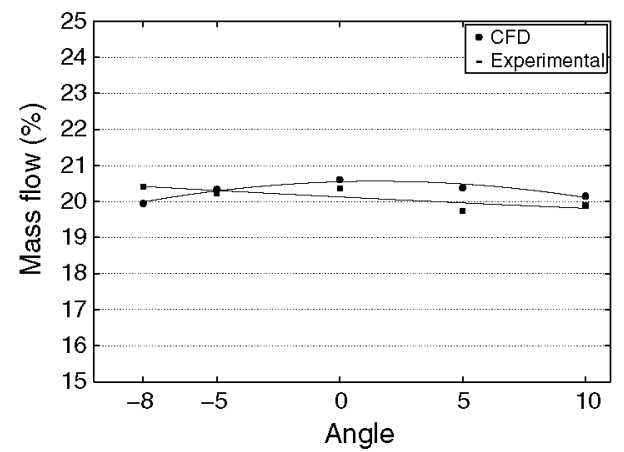

(c) Level III

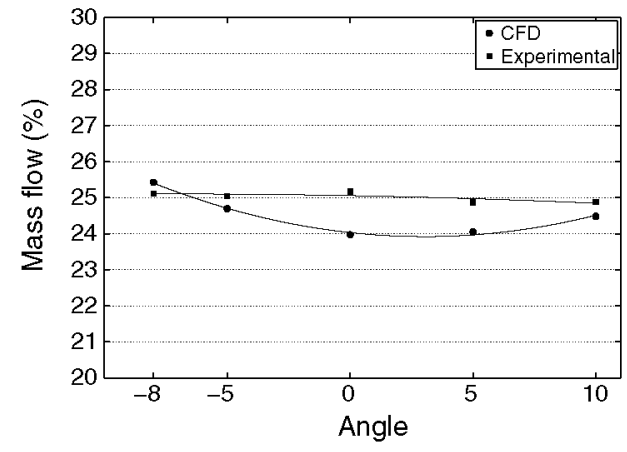

(b) Level II

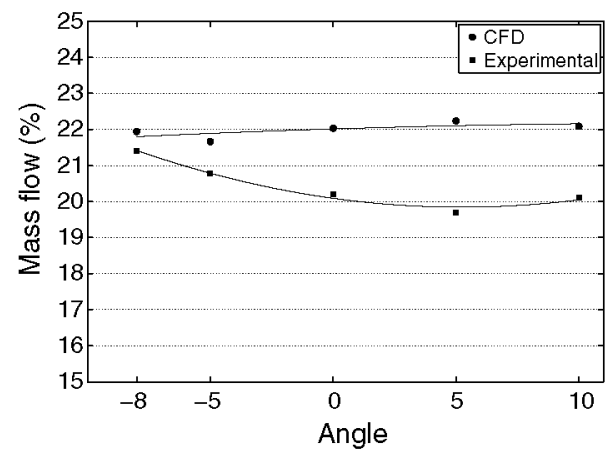

(d) Level IV

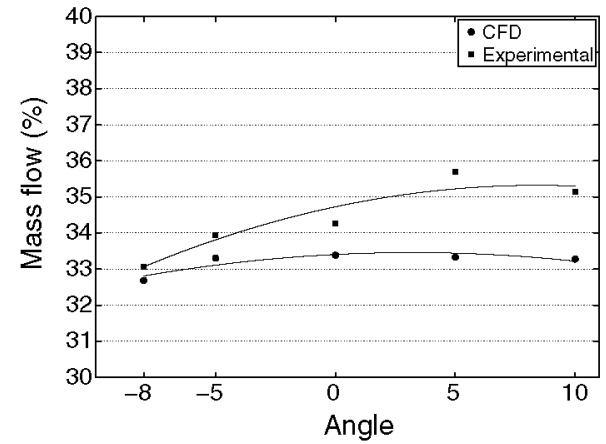

Fig. 9. Distribution of the gas mass flow rate according to the burner levels.

describe the collisions between particles if it was the case. The particle momentum parallel and perpendicular to the wall could be reduced by coefficients of restitution (constant values of 1 and 0.9 , respectively,

(a) Level I

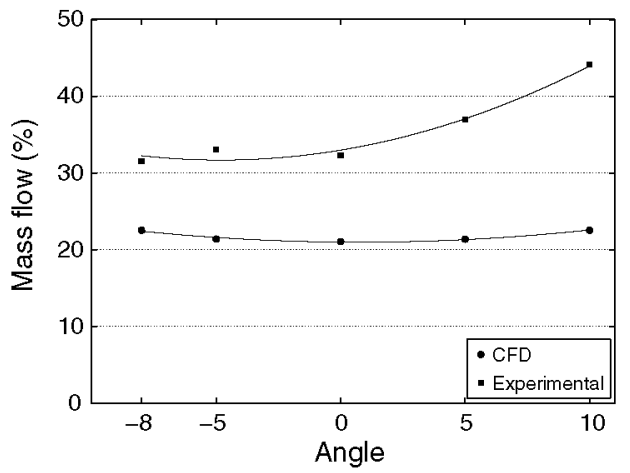

(c) Level III

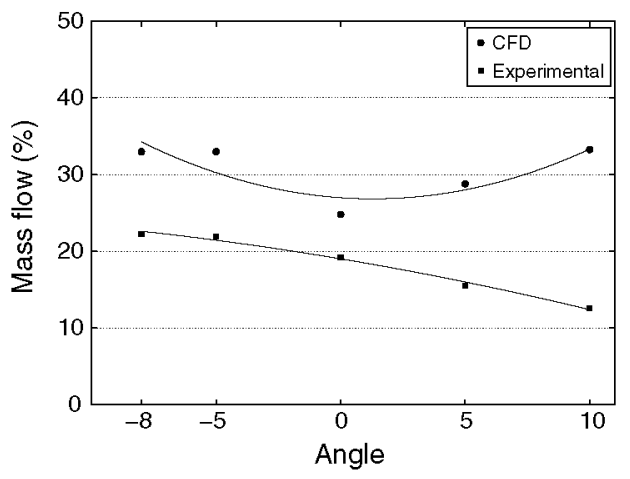

will be used in all the simulations) and the perpendicular one is reversed. Furthermore, the effects of the shape of the particles and the roughness and material of the wall, as well as the erosion process, are not considered

\section{(b) Level II}

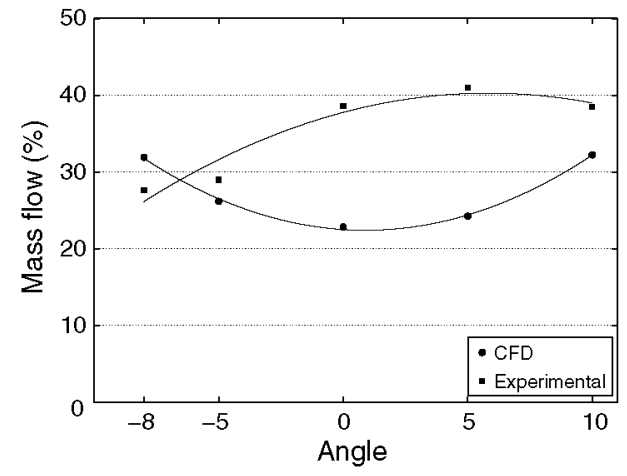

(d) Level IV

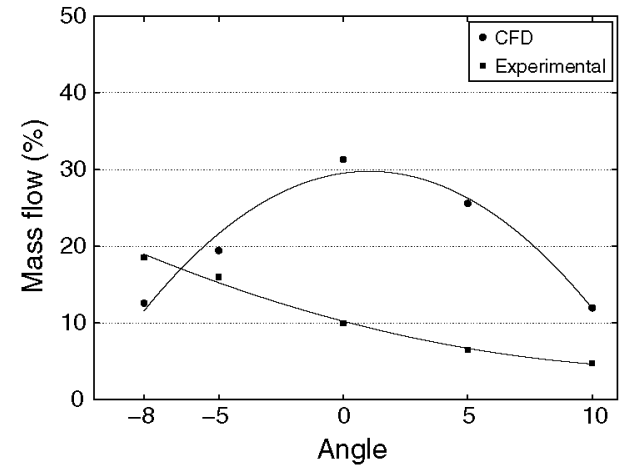

Fig. 10. Distribution of the coal mass flow rate according to the burner levels. 
in the simulations. For additional details related with particle-wall collisions Sommerfeld and Huber [20], and references therein, can be seen.

\section{Numerical results and validation with plant data}

As mentioned in Section 2, several tests have been carried out in the power plant for different positions of the concentrator. In order to validate the numerical results obtained for each angle of the concentrator, we will compare them with the averaged values obtained in all the tests for that position.

The gas velocity in the plane $Y=0$ is plotted in Fig. 4 for each position of the concentrator. It can be observed that the mean velocity, therefore the mass flow rate, increases as the level does. Furthermore, such similar results indicate that the position of the concentrator should not affect the gas flow rate distribution. These facts are illustrated in Fig. 5 where a comparison between the simulated and measured flow rate distributions for each position of the concentrator is presented.

As we are dealing with a turbulent flow, in addition to the gas velocity, the distribution of the coal mass flow is strongly influenced by the turbulent dispersion of particles, as discussed in Section 2. Thus, seeing the turbulent viscosity in Fig. 6, we can have an idea of where are the most turbulent areas in the ducts and therefore where more particles are dispersed. Therefore in general it can expected that through levels II and III the amount of coal is higher in all the concentrator positions. Fig. 7 shows mean particle trajectories colored by its diameter in each of the cases considered. Although some random trajectories are only displayed (the same particles are painted in all figures), these figures can give us an idea of how the particles are dispersed inside the mill ducts. So for example we can see that fewer particles reach level IV at positions -8 and 10 or that the particle distribution at positions -5 and 5 is similar but the latter is slightly more even. Fig. $6(\mathrm{c})$ indicates that when the concentrator is not inclined, the flow in the central tube is less turbulent than in the other cases and a high percentage of particles reaches the upper level as we can see in Fig. 7(c).

In Fig. 8, with blue dots, we can see the predicted distribution among the different levels of the mass of coal depending on the position of the concentrator and the corresponding experimental measurements are represented with red squares. In Figs. 5 and 8 error bars are plotted in the experimental values to give an indication of the variation between the different measurements, with the average value shown by the symbol. Looking at these error bars, the considerable uncertainty in the experimental data concerning the coal flow can be observed. Fig. 5 shows a very good agreement between the predicted results and the experimental ones. The experimental data show very little variation between the different tests, so the error bars are very small and hardly visible. This fact indicates the reliability of the type of measurements performed for the gas, in contrast to those carried out for the pulverized coal.

As mentioned before, the predicted percentage of coal mass is higher for levels II and III in all positions, exception made for position 0 , and this uneven distribution is much more pronounced the greater the inclination of the concentrator is, as can be seen in Fig. 8 .

The representativeness of the samples is strongly influenced by the location of the sampling plane and the number of sampling points. It is well-known that the sampling plane must be located in a section of straight duct (preferably vertical) with a shape and a constant cross section and as far away as possible from any previous or subsequent disturbance, which could produce a change in flow direction. Furthermore, the gas stream in the conduit at the location of the sampling plane must have a sufficiently constant speed. High pulverized coal flows in the ducts introduce difficulties for obtaining representative samples.

In the case of As Pontes Power Plant besides the samples corresponding to the four inlet ducts of coal to the furnace, additional sampling is performed in the conduit before the coal concentrator. These measures correspond to the total coal introduced in the burner and are considered more representative because of the situation of the sampling plane in a straight and vertical section of the mill-duct.

Finally, Figs. 9 and 10 plot the gas and coal flow rates obtained at each level for the five positions considered. The tendency of coal flow obtained in the experiments is always monotone (increasing or decreasing) while the predicted results show a symmetrical behavior. Taking into account the position of the concentrator within the system, it can be expected that the results of the distribution are similar for the opposite angles.

\section{Conclusions}

Simulations were used to predict the distribution of the gas and coal flow rates in the mill-duct system of an operating Power Station. Good agreement between the simulated and experimental results was obtained for those related to the gas. Concerning the coal flow, the results provided by CFD differ significantly from the experimental ones. The calculated results are more consistent with the expected symmetrical behavior when varying the positions of the concentrator. This is due to the fact that the technique used to obtain the measurements is not precise. We may therefore conclude that the simulations are essential for the Thermal Power Station, to understand the distribution of coal and gas flow rates in the mill-duct system because of the difficulty of performing direct measurements on the same.

Directive 2010/75/EC of the European Parliament and of the Council of 24 November 2010 on industrial emissions (integrated pollution prevention and control) set to combustion plants with capacities greater than 50 MWth new Emission Limit Values (ELVs), applicable from January 1,2016 , more restrictive than those covered by Directive 2001/80/EC on the limitation of certain pollutants into the air from large combustion plants.

Primary measures to reduce $\mathrm{NO}_{x}$ currently available in the As Pontes Power Plant do not achieve emission levels below $200 \mathrm{mg} / \mathrm{m}^{3} \mathrm{~N}$, limit value in the new directive, so it will be necessary to implement additional measures.

CFD modeling of the mill-burner systems and of the combustion in the furnace will provide relevant information for the redesign, together with experimental tests in the current situation of the industrial facility.

\section{Acknowledgments}

This work was partially supported by the Spanish MICINN projects ENE2005-09190-C04-01/CON and MTM2008-02483. It was also supported by the Fundación Ciudad de la Energía under project SIMULOX and by the MEC under the CONSOLIDER project i-MATH.

\section{References}

[1] A.D. Jensen, G. Elsen, Reducing unburned carbon using coal flow distribution analysis, In: 2003 Conference on Unburned Carbon on Utility Fly Ash, 28th October, 2003.

[2] Y. Yan, Mass flow measurement of bulk solid in pneumatic pipelines, Measurement Science and Technology 7 (12) (1996) 1687-1706.

[3] C. Arakaki, A. Ghaderi, B.K. Datta, B. Lie, Non-intrusive mass flow measurements in pneumatic transport, In: CHoPS-05, 2006 the 5th International Conference for Conveying and Handling of Particulate Solids, Sorrento, Italy, 27th-30th August, 2006.

[4] W.H. Ahmed, B.I. Ismail, Innovative Techniques for Two-phase Flow Measurements, Recent Patents on Electrical Engineering, Bentham Science Publishers Ltd., 2008.

[5] Y. Zheng, Q. Liu, Review of techniques for the mass flow rate measurement of pneumatically conveyed solids, Measurement 44 (4) (2011) 589-604.

[6] K.V. Shah, R. Vuthaluru, H.B. Vuthaluru, CFD based investigations into optimization of coal pulveriser performance: effect of classifier vane settings, Fuel Processing Technology 90 (9) (2009) 1135-1141.

[7] D. Dodds, J. Naser, J. Staples, C. Black, L. Marshall, V. Nightingale, Experimental and numerical study of the pulverised-fuel distribution in the mill-duct system of the Loy Yang B lignite fuelled power station, Powder Technology 207 (1-3) (2011) 257-269.

[8] H.B. Vuthaluru, V.K. Pareek, R. Vuthaluru, Multiphase flow simulation of a simplified coal pulveriser, Fuel Processing Technology 86 (11) (2005) 1195-1205. 
[9] R. Vuthaluru, O. Kruger, M. Abhishek, V.K. Pareek, H.B. Vuthaluru, Investigation of wear pattern in a complex coal pulveriser using CFD modelling, Fuel Processing Technology 87 (8) (2006) 687-694.

[10] C. Arakaki, A. Ghaderi, A. Sather, C. Ratnayake, G.G. Enstad, Air mass balance for mass flow rate calculation in pneumatic conveying, Powder Technology 202 (1-3) (2010) 62-70.

[11] T.-H. Shih, W.W. Liou, A. Shabbir, Z. Yang, J. Zhu, A new $k-\varepsilon$ eddy viscosity model for high Reynolds number turbulent flows, Computers \& Fluids 24 (3) (1995) $227-238$.

[12] L. Schiller, Z. Naumann, Fundamental calculations in gravitational processing, Zeitschrift des Vereines Deutscher Ingenieure 77 (1933) 318-320.

[13] A.D. Gosman, E. Ioannides, Aspects of computer simulation of liquid-fueled combustors, AIAA Journal of Energy 7 (6) (1983) 482-490.

[14] J.M. Macinnes, F.V. Bracco, Stochastic particle dispersion modeling and the tracer-particle limit, Physics of Fluids 12 (1992) 2809-2824.
[15] E.A. Hennick, M.F. Lightstone, A comparison of stochastic separated flow models for particle dispersion in turbulent flows, Energy \& Fuels 14 (2000) 95-103.

[16] J. Pozorski, J.-P. Minier, On the Lagrangian turbulent dispersion models based on the Langevin equation, International Joumal of Multiphase Flow 24 (1998) 913-945.

[17] J. Pozorski, J.-P. Minier, Probability density function modeling of dispersed two-phase turbulent flows, Physical Review E 59 (1) (1999) 855-863.

[18] E. Peirano, S. Chibbaro, J. Pozorski, J.-P. Minier, Mean-field/PDF numerical approach for polydispersed turbulent two-phase flows, Progress in Energy and Combustion Science 32 (2006) 315-371.

[19] N. Mitarai, H. Nakanishi, Hard-sphere limit of soft-sphere model for granular materials: stiffness dependence of steady granular flow, Physical Review E 67 (2003) 021301.

[20] M. Sommerfeld, N. Huber, Experimental analysis and modelling of particle-wall collisions, International Journal of Multiphase Flow 25 (6-7) (1999) 1457-1489. 